\title{
Quality of Ribonucleic Acid Extraction for Real-Time Reverse Transcription-PCR (rRT-PCR) of SARS-CoV-2: Importance of Internal Control Monitoring
}

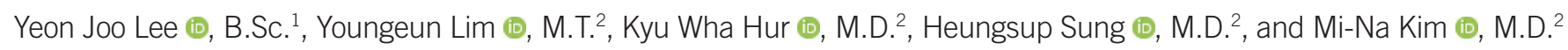
${ }^{1}$ Konkuk University School of Medicine, Seoul, Korea; ${ }^{2}$ Department of Laboratory Medicine, University of Ulsan, College of Medicine and Asan Medical Center, Seoul, Korea

Dear Editor,

Severe acute respiratory syndrome coronavirus 2 (SARS-CoV-2) emerged as a novel virus causing pneumonia in December 2019 in Wuhan city, Hubei province, China, which led to a huge outbreak of the disease, now widely known as COVID-19, spreading to 46 countries by 26th February 2020 [1]. The WHO recommends nucleic acid amplification tests for laboratory confirmation of COVID-19 [2]. Over nine days, from 18th-26th February 2020, approximately 50,000 tests for suspected cases of COVID-19 were performed in Korea, resulting in a diagnosis of 1,742 new cases in this period [3, 4]. On 6th February 2020, Emergency Use Authorization (EUA) for COVID-19 testing was implemented in Korea, permitting rapid expansion of capacity in clinical and public health laboratories [5]. Such a surge in demand for laboratory diagnostic tests inevitably results in insufficient validation of new tests accompanied by lack of resources.

On 17th February 2020, real-time reverse transcription ( $r R T$ )PCR was set up for SARS-CoV-2 detection by Allplex 2019-nCoV assay (Seegene, Seoul, Korea) using the CFX96 Touch Real-Time PCR Detection System (Bio-Rad, Hercules, CA, USA) in the clinical laboratory in Asan Medical Center, Seoul, Korea. However, we experienced cycle threshold (Ct) prolongation of the internal control (IC) and therefore investigated the cause. The Institutional Review Board of Asan Medical Center (IRB No. 20200370) exempted the study from further review and the need for informed consent.

Sputum samples collected without preservation and nasopharyngeal (NP) samples collected using flocked swabs were transported in a UTM Viral Transport system (Copan, Brescia, Italy). Each sample was first spiked with the IC in Allplex kit, and then RNA was extracted with the automated silica-coated magnetic bead system of Real-Prep (BioSewoom Inc., Seoul, Korea) using Real-Prep Viral DNA/RNA kit (BioSewoom). This system was first introduced in the laboratory on 14th February 2020 for SARSCoV-2 rRT-PCR. For rRT-PCR, $8 \mu \mathrm{L}$ of the RNA extract was manually added to PCR mixtures in Allplex kit, and Ct values of IC
Received: March 12, 2020

Revision received: April 6, 2020

Accepted: May 19, 2020

Corresponding author: Mi-Na Kim, M.D.

Department of Laboratory Medicine, University of Ulsan, College of Medicine and Asan Medical Center, 88 Olympic-ro 43 gil, Songpa-gu, Seoul 05505, Korea

Tel: +82-2-3010-4511, Fax: +82-2-478-0884, E-mail: mnkim@amc.seoul.kr

\section{(i) (3)}

\section{(c) Korean Society for Laboratory Medicine}

This is an Open Access article distributed under the terms of the Creative Commons Attribution Non-Commercial License (https://creativecommons.org/licenses/by-nc/4.0) which permits unrestricted non-commercial use, distribution, and reproduction in any medium, provided the original work is properly cited.
Co-corresponding author: Heungsup Sung, M.D.

Department of Laboratory Medicine, University of Ulsan, College of Medicine and Asan Medical Center, 88 Olympic-ro 43 gil, Songpa-gu, Seoul 05505, Korea

Tel:+82-2-3010-4499, Fax: +82-2-478-0884, E-mail: sung@amc.seoul.kr 
were monitored for all samples. Ninety-four runs comprising 3,345 tests were performed over nine days from 18th-26th February 2020. There were no positive clinical samples. The average Ct value of IC was $22.60 \pm 0.99$ at the first run, which increased to a maximum of $34.97 \pm 2.99$ among the runs performed on 26th February 2020 for NP samples; a similar increase from $23.94 \pm 1.48$ to 38.67 was detected for sputum samples (Fig. 1). There was an increased failure in amplification of IC during the same period, with a maximum failure rate of $41.2 \%$ for NP and $88.9 \%$ for sputum samples on 26th February 2020 (Table 1).

To evaluate the performance of the RNA extraction step, 31 and 35 samples were prepared using the Real-Prep-in-use and the new Real-Prep system, respectively, and all 66 eluates were submitted to the same run of rRT-PCR. The IC failure rate of NP samples was $51.6 \%$ with an average $\mathrm{Ct}$ value of $35.4 \pm 2.00$ using Real-Prep-in-use, but it was $0 \%$ with a Ct value of $30.24 \pm 1.77$ using the new Real-Prep system (Fig. 1). Examination of the Real-Prep-in-use by the manufacturer revealed that all magnetic rods were coated with a substantial number of magnetic beads, which would hamper the ability to hold magnetic beads. Therefore, Ct prolongation and failure of IC were attributed to
Table 1. Failure rates of IC for SARS-CoV-2 rRT-PCR for nine days in February 2020

\begin{tabular}{lccc}
\hline \multirow{2}{*}{ Date_run* } & \multicolumn{3}{c}{$N$ failures $/ \mathrm{N}$ tests (\%) } \\
\cline { 2 - 4 } & NP swab & Sputum & Total \\
\hline $18 \_1$ & $0 / 13(0)$ & $0 / 11(0)$ & $0 / 24(0)$ \\
$19 \_1$ & $0 / 22(0)$ & $0 / 21(0)$ & $0 / 43(0)$ \\
$20 \_1$ & $0 / 31(0)$ & $1 / 34(2.9)$ & $1 / 65(1.5)$ \\
$21 \_1$ & $0 / 15(0)$ & $0 / 16(0)$ & $0 / 31(0)$ \\
$22 \_1$ & $0 / 24(0)$ & $0 / 5(0)$ & $0 / 29(0)$ \\
$23 \_1$ & $0 / 15(0)$ & $1 / 10(10)$ & $1 / 25(4)$ \\
$24 \_1$ & $1 / 19(5.3)$ & $0 / 13(0)$ & $1 / 32(3.1)$ \\
$25 \_1$ & $0 / 21(0)$ & $0 / 11(0)$ & $0 / 32(0)$ \\
$26 \_1$ & $0 / 20(0)$ & $0 / 17(0)$ & $0 / 37(0)$ \\
$26 \_5$ & $19 / 47(40.4)$ & $11 / 17(64.7)$ & $30 / 64(46.9)$ \\
$26 \_9$ & $7 / 17(41.2)$ & $8 / 9(88.9)$ & $15 / 26(57.7)$ \\
26_in_use* & $7 / 21(33.3)$ & $9 / 10(90)$ & $16 / 31(51.6)$ \\
26_new ${ }^{\dagger}$ & $0 / 28(0)$ & $0 / 7(0)$ & $0 / 35(0)$ \\
\hline
\end{tabular}

*RNA of all runs was extracted with the Real-Prep in use (Biosewoom, Seoul, Korea) including Feb 26_in_use except ${ }^{\dagger} F e b$ 26_new, for which RNA was extracted with the new Real-Prep system.

Abbreviations: IC, internal control; rRT-PCR, real-time reverse transcription PCR; SARS-CoV-2, severe acute respiratory syndrome coronavirus 2; NP, nasopharyngeal.

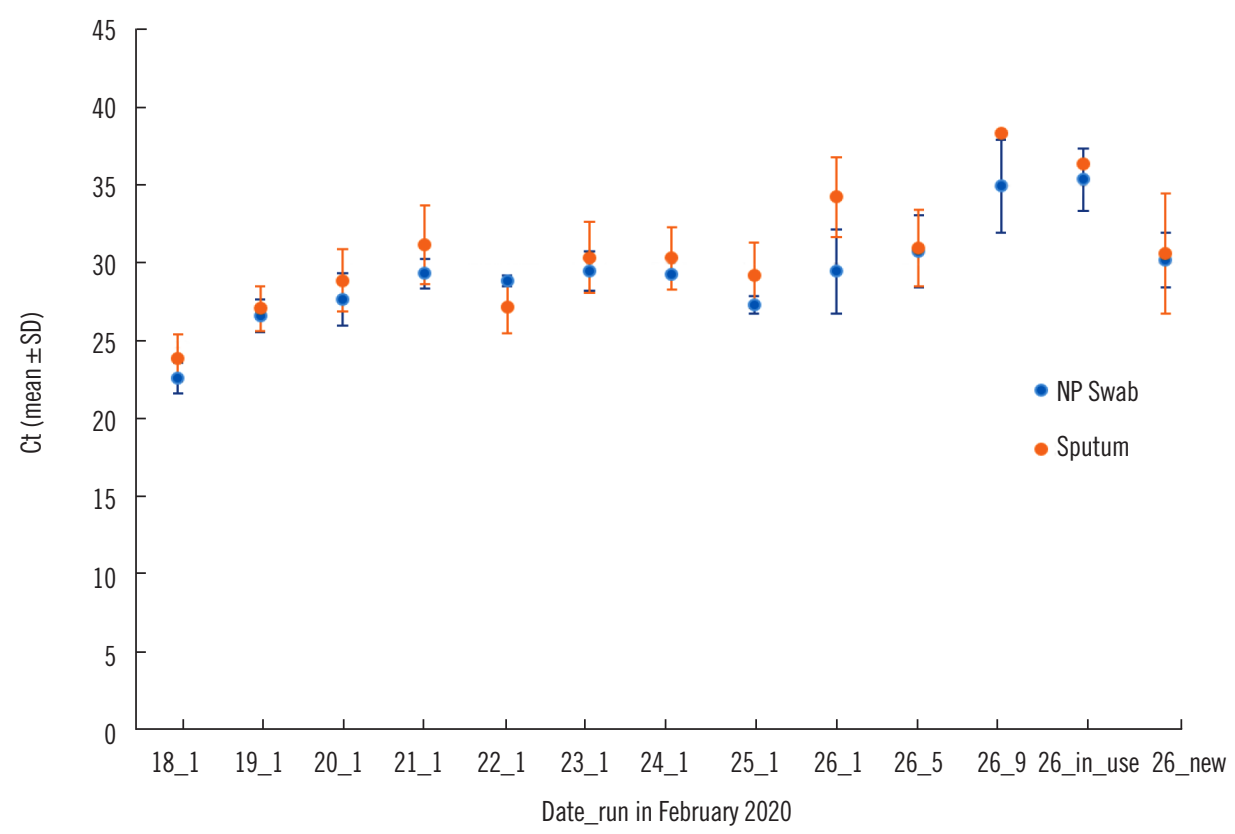

Fig. 1. Trends of Ct values of the IC in rRT-PCR for SARS-CoV-2 detection. At the end of 26th February 2020, in 31 samples, RNA was extracted using the Real-Prep (BioSewoom, Seoul, Korea) system in use, 2-26 (in use), and in 35 samples, RNA was extracted using the new Real-Prep system, 2-26 (new); all of these were submitted to a single run of PCR for comparison.

Abbreviations: Ct, cycle threshold; IC, internal control; rRT-PCR, real-time reverse transcription PCR; SARS-CoV-2, severe acute respiratory syndrome coronavirus 2; NP, nasopharyngeal. 
the poor quality of the RNA extraction step.

The purpose of $\mathrm{IC}$ is to validate nucleic acid extraction and the efficiency of PCR [6]. For this purpose, housekeeping genes intrinsic to samples are used as IC, or artificial IC can be spiked in the samples before preparation. Therefore, a well-designed IC is an essential component of PCR kits to detect viral target genes [7]. This is particularly relevant for EUA-approved kits, which are exempt from clinical validation requirements. Although five SARS-CoV-2 rRT-PCR have been approved by the EUA as of 23rd March 2020 in Korea [8], RNA bacteriophage as an IC is available with the Allplex kit only, monitoring the entire process from RNA extraction to PCR (http://www.seegene.com/assays/allplex_2019_ncov_assay). The analytical performance of PCR is largely dependent on the quality of the sample and nucleic acid extraction efficiency [9]. We found more prominent deterioration in PCR efficiency of IC with sputum samples than with NP samples. Both sputum and NP samples were recommended with same cut-off of $\mathrm{Ct}$ value $<40$ by the manufacturer, but sputum samples require homogenization, which critically influences the efficiency to extract nucleic acids and eliminate interferents that can inhibit PCR [7, 9]. Contamination of magnetic rods is an additional concern that could lead to false positivity, although no positive samples were detected during this investigation.

As the experience of the Middle East Respiratory Syndrome outbreak of 2015 in Korea, an epidemic of emerging infectious diseases necessitates clinical laboratories to conduct new tests at a large scale in a short period of time with kits and equipment that have not been thoroughly validated [10]. This situation poses a risk affecting the quality of the diagnostic tests approved by the EUA. Accordingly, EUA-approved kits should include adequate ICs to allow for monitoring of the overall analytical performance. Clinical microbiologists should closely monitor the ICs for each run with products under an EUA condition.

\section{ACKNOWLEDGEMENTS}

None.

\section{AUTHOR CONTRIBUTIONS}

MNK and HS led the entire study from ideation to writing this paper. YJL mainly wrote this paper. YJL conducted the experiments and KWH analyzed the data.

\section{CONFLICTS OF INTEREST}

The authors declare no conflict of interest.

\section{RESEARCH FUNDING}

None declared.

\section{ORCID}

Yeon Joo Lee

Youngeun Lim

Kyu Wha Hur

Heungsup Sung

Mi-Na Kim

https://orcid.org/0000-0002-8066-0724
https://orcid.org/0000-0001-9000-6809
https://orcid.org/0000-0002-2159-3592
https://orcid.org/0000-0002-6062-4451
https://orcid.org/0000-0002-4624-6925

\section{REFERENCES}

1. World Health Organization. Coronavirus disease 2019 (COVID-19) Situation Report-38. https://www.who.int/docs/default-source/coronaviruse/ situation-reports/20200227-sitrep-38-covid-19.pdf?sfvrsn=9f98940c_4 (Updated on 27 Feb 2020).

2. World Health Organization. Laboratory testing for coronavirus disease 2019 (COVID-19) in suspected human cases. Interim guidance. https:// apps.who.int/iris/handle/10665/331329 (Updated on 2 Mar 2020).

3. Korea Centers for Disease Control and Prevention. The 15 new cases of COVID-19 have been confirmed in Korea. https://www.cdc.go.kr/board/ board.es?mid=a30402000000\&bid=0030\&act=view\&list_no=366239 \&tag=\&nPage $=1$ (Updated on 19 Feb 2020).

4. Korea Centers for Disease Control and Prevention. The Updates of CO VID-19 in Republic of Korea as of 26 February, 2020. https://www.cdc. go.kr/board/board.es?mid=a30402000000\&bid=0030\&act=view\&list no=366350\&tag=\&nPage $=1$ (Updated on 26 Feb 2020).

5. JS Park, YS Choi, Yoo CK. Emergency use authorization of in-vitro diagnostics for infectious disease. Public Health Weekly Report 2017;10: 555-9.

6. CLSI. Quantitative molecular methods for infectious disease. Approved guideline. 2nd ed. CLSI MM06-A2. Wayne, PA: Clinical and Laboratory Standards Institute. 2010.

7. Kim MN, Ko YJ, Seong MW, Kim JS, Shin BM, Sung H. Analytical and clinical validation of six commercial Middle East Respiratory Syndrome coronavirus RNA detection kits based on real-time reverse-transcription PCR. Ann Lab Med 2016;36:450-6.

8. Kim YJ, Sung H, Ki CS, Hur M. COVID-19 testing in South Korea: current status and the need for faster diagnostics. Ann Lab Med 2020;40: 349-3509.

9. Sung H, Yong D, Ki CS, Kim JS, Seong MW, Lee H, et al. Comparative evaluation of three homogenization methods for isolating Middle East Respiratory Syndrome coronavirus nucleic acids from sputum samples for real-time reverse transcription PCR. Ann Lab Med 2016;36:457-62.

10. Lee MK, Kim S, Kim MN, Kweon OJ, Lim YK, Ki CS, et al. Survey of clinical laboratory practices for 2015 Middle East Respiratory Syndrome coronavirus outbreak in the Republic of Korea. Ann Lab Med 2016;36: 154-61. 